\title{
The factorization of simple knots
}

\author{
By E. BAYER, J. A. HILLMAN*† AND C. KEARTON† \\ University of Geneva and University of Durham
}

(Received 19 December 1980)

Abstract. For high-dimensional simple knots we give two theorems concerning unique factorization into irreducible knots, and provide examples to show that the hypotheses are necessary in each case.

\section{Introduction}

The purpose of this paper is to collate and extend the known results on the factorization of high dimensional knots. By an $n$-knot we mean an oriented smooth or locally flat PL pair $\left(S^{n+2}, \Sigma^{n}\right)$, where $\Sigma^{n}$ is homeomorphic to the $n$-sphere $S^{n}$. The sum $k+l$ of two $n$-knots $k$ and $l$ is obtained by excising the interior of a tubular neighbourhood of a point on each $\Sigma^{n}$ and identifying the boundaries of the resulting knotted ball pairs so that the orientations match up. A knot $k$ is irreducible if it cannot be written as the sum of two non-trivial knots. It is a result of $H$. Schubert (16) that for $n=1$, every knot factorizes into finitely many irreducibles, and that factorization is unique (up to the order of the factors).

Given an $n$-knot $k$, the exterior $K$ is the closed complement of a tubular neighbourhood of $\Sigma^{n}$. The knot $k$ is simple if $K$ has the homotopy [(n-1)/2]-type of a circle; that is $\pi_{1}(K) \cong \pi_{1}\left(S^{1}\right)$ for $1 \leqslant i \leqslant(n-1) / 2$. For $n \geqslant 3$, this is the most that can be asked without making $k$ trivial (see $(11,12)$ ). The knot $k$ is fibred if $K$ is fibred over the circle, and we let $\widetilde{K}$ denote the infinite cyclic cover of $K$.

In Section 1 we give a short proof that every simple $n$-knot, $n \geqslant 3$, factorizes into finitely many irreducibles. A more general result was published by A. B. Sosinskii in (18), but note the assertion of T. Maeda in (14).

Let $k$ be a simple $(2 q-1)$-knot, $q \geqslant 2$. There are two ways of classifying such knots in terms of algebraic invariants. The first of these, due to J. Levine, is in terms of the $S$-equivalence class of the Seifert matrix of $k$; details may be found in (12). The second method uses the Blanchfield duality pairing, $\langle\rangle:, H_{q}(\widetilde{K}) \times H_{q}(\tilde{K}) \rightarrow \Lambda_{0} / \Lambda$, where $\Lambda=\mathbb{Z}\left[t, t^{-1}\right], \Lambda_{0}$ is the field of fractions of $\Lambda$, and $H_{q}(\tilde{K})$ is regarded as a $\Lambda$-module. Details of this method may be found in $(7,8,20,21)$.

Each such knot $k$ has associated with it a quadratic form, as outlined in Section 2. If this form is definite, then $k$ is said to be definite. The knot $k$ is fibred if and only if the leading coefficient of its Alexander polynomial is \pm 1 ; this follows easily from the results of R. H. Crowell (3) and W. Browder and J. Levine(2). In Section 2 we show

* Supported by a Research Grant from the Science Research Council of Great Britain.

$\dagger$ Recipients of a European Short Visit Grant from the Science Research Council of Great Britain. 
that for $q \geqslant 3$, every fibred definite knot factorizes uniquely into irreducibles. Sections 3-6 are devoted to showing that each of the hypotheses $q \geqslant 3$, fibred, and definite are necessary for this result.

Next we turn our attention to simple $2 q$-knots, $q \geqslant 4$, for which $H_{q}(\tilde{K})$ is finite of odd order. Such knots have been classified by $S$. Kojima (10) in terms of a quadratic pairing $[]:, H_{q}(\tilde{K}) \times H_{q}(\tilde{K}) \rightarrow \mathbb{Q} / \mathbb{Z}$ together with an isometry $t$; the pair $([], t$,$) is called$ the Levine pairing of $k$. In Section 7 we outline a unique factorization theorem for a certain subclass of these knots, details of which appear in (6), and in Section 8 we give examples to show that factorization is not in general unique.

\section{Finite factorization of simple knots}

Let $k$ be a simple $n$-knot and define $g(k)$ in the following way. If $n=2 q-1$, then $g(k)=\operatorname{dim}_{\mathbb{Q}} H_{q}(\widetilde{K} ; \mathbb{Q})$. If $n=2 q$, let $T_{q}(\widetilde{K})$ denote the $\mathbb{Z}$-torsion submodule of $H_{q}(\widetilde{K})$; by a result of M. A. Kervaire (9), $T_{q}(\widetilde{K})$ is finite of order $\left|T_{q}(\widetilde{K})\right|$. We set

$$
g(k)=\operatorname{dim}_{\mathbb{Q}} H_{q}(\widetilde{K} ; \mathbb{Q}), h(k)=\left|T_{q}(\tilde{K})\right| .
$$

Theorem 1.1. Let $k$ be a simple $n$-knot, $n \geqslant 3$. Then $k$ factorizes into finitely many irreducible knots.

Proof. If $n=2 q-1$, then $g(k)=0 \Leftrightarrow H_{q}(\tilde{K} ; \mathbb{Q})=0 \Leftrightarrow H_{q}(\tilde{K} ; \mathbb{Z})=0$, since the latter is $\mathbb{Z}$-tórsion-free (see $(7)) \Leftrightarrow k$ is unknotted (see $(7,8)$ ).

If $n=2 q$, then $g(k)=0$ and $h(k)=1 \Leftrightarrow H_{q}(\tilde{K} ; \mathbb{Q})=0$ and

$$
T_{q}(\tilde{K})=0 \Leftrightarrow H_{q}(\tilde{K} ; \mathbb{Z})=0 \Leftrightarrow H_{q}(\tilde{K} ; \mathbb{Z})=0=H_{q+1}(\tilde{K} ; \mathbb{Z}) \Leftrightarrow K
$$

is a homotopy circle $\Leftrightarrow k$ is unknotted (see (11)).

Furthermore, it is clear that $g(k+l)=g(k)+g(l)$ and $h(k+l)=h(k) h(l)$.

The result follows at once.

\section{Unique factorization of fibred definite simple $(2 q-1)-k n o t s, q \geqslant 3$}

Let $k$ be a simple $(2 q-1)$-knot, and let $A$ be a Seifert matrix of $k$. By a result of Trotter(19), $A$ is $S$-equivalent to a non-singular matrix, and so we may assume that $A$ is non-singular. Furthermore, any non-singular matrix which is $S$-equivalent to $A$ is congruent to $A$ over the rational numbers. Set $S=A+A^{\prime}, T=A^{-1} A^{\prime}$, and note that $T^{\prime} S T=A A^{\prime-1}\left(A+A^{\prime}\right) A^{-1} A^{\prime}=A^{\prime}+A=S$. If $A_{1}=P^{\prime} A P$, then

$$
S_{1}=A_{1}+A_{1}^{\prime}=P^{\prime} S P \text {, and } T_{1}=A_{1}^{-1} A_{1}^{\prime}=P^{-1} T P .
$$

Thus $k$ determines a quadratic space $V$ together with an isometry $\tau$, represented by the pair $(S, T)$. The knot is definite if $V$ is definite.

If $k$ is fibred, then $\operatorname{det} A= \pm 1$, since $\operatorname{det} A$ is the leading coefficient of the Alexander polynomial $\operatorname{det}\left(t A+(-1)^{a} A^{\prime}\right)$. If $q \geqslant 3$, then the converse is true by the results of R. H. Crowell (4) and W. Browder and J. Levine (2). Moreover, any non-singular matrix which is $S$-equivalent to $A$ is congruent to $A$ over the integers. Thus a fibred knot gives rise to a quadratic lattice $L$ and isometry $\tau$ represented by $(S, T)$. 
Note that

$$
S=A+A^{\prime}=A+A T=A(I+T)
$$

so $A=S(I+T)^{-1}$. Thus given $(S, T)$ we can recover the Seifert matrix $A$.

By the results of J. Levine (12), the isotopy class of $k$ determines and is determined by the $S$-equivalence class of $A$, when $q \geqslant 2$. If $k$ is fibred, this reduces to the integral congruence class of $A$ (where $A$ is non-singular).

THEOREM $2 \cdot 1$. Let $k$ be a fibred definite simple $(2 q-1)$-knot, $q \geqslant 3$. Then $k$ factorizes uniquely into irreducible knots.

Proof. Let $L$ be the quadratic lattice of $k$. Since $L$ is definite, it has a unique orthogonal decomposition into indecomposable sublattices by a theorem of Eichler (see (3), p. 363). Say $L=L_{1} \perp \ldots \perp L_{r}$. Now $L$ can be regarded as a $\Lambda$-module via the action of the isometry $\tau$. (Recall that $\Lambda=\mathbb{Z}\left[t, t^{-1}\right]$.) But $L=\tau L=\tau L_{1} \perp \ldots \perp \tau L_{r}$ is another orthogonal splitting of $L$ into indecomposable sublattices, so the action of $t$ is to permute the $L_{i}$. Thus $L$ splits orthogonally as $L=L_{1}^{\prime} \perp \ldots \perp L_{m}^{\prime}$, where each $L_{i}^{\prime}$ is a $\Lambda$-module which is irreducible in the sense that it cannot be written as the orthogonal sum of two non-trivial $\Lambda$-modules. Moreover, this splitting is unique.

Choosing $\mathbb{Z}$-bases of each $L_{i}^{\prime}$ we can assume that $S, T$ have block diagonal form:

and hence

$$
S=\left(\begin{array}{lll}
S_{1} & & \\
& \ddots & \\
& & S_{m}
\end{array}\right), \quad T=\left(\begin{array}{ccc}
T_{1} & & \\
& \ddots & \\
& & T_{m}
\end{array}\right)
$$

also has block diagonal form. Now

$$
A=\left(\begin{array}{lll}
A_{1} & & \\
& \ddots & \\
& & A_{m}
\end{array}\right)
$$

$$
\operatorname{det}\left(A+(-1)^{q} A^{\prime}\right)=\prod_{i=1}^{m} \operatorname{det}\left(A_{i}+(-1)^{q} A_{i}^{\prime}\right),
$$

and so for each $i$ we see that $A_{i}+(-1)^{q} A_{i}^{\prime}$ is unimodular. Thus by (12), $A_{i}$ is a Seifert matrix of a simple $(2 q-1)$-knot $k_{i}$, and $k=k_{1}+\ldots+k_{m}$. Because $L_{i}^{\prime}$ is unique and indecomposable, $k_{i}$ is unique and irreducible, and the result is proved. I

3. Non-unique factorization of fibred simple $(2 q-1)$-knots $), q \geqslant 3$

We shall reformulate the proof of [(1); Section 1] using Blanchfield forms instead of Seifert matrices. There is a bijection between the isotopy classes of simple $(2 q-1)$ knots, $q \geqslant 3$, and the isometry classes of $(-1)^{q+1}$-Blanchfield forms (see $(7,8)$ ). Therefore it suffices to prove that factorization is not unique for $(-1)^{q+1}$-Blanchfield forms.

Let $\lambda \in \mathbb{Z}[t]$ be such that $\lambda(1)= \pm 1, \lambda(t)=t^{\operatorname{deg} \lambda} \lambda\left(t^{-1}\right)$. Let $R=\Lambda /(\lambda)=\mathbb{Z}\left[\tau, \tau^{-1}\right]$, where $\tau$ is a root of $\lambda$. Define an involution $\bar{t}=t^{-1}$ in $\Lambda$, which induces the involution $\bar{\tau}=\tau^{-1}$ in $R$.

Let $H$ be the standard hyperbolic (+1)-Blanchfield form:

$$
\begin{gathered}
H:(\operatorname{Re} \oplus R f) \times(\operatorname{Re} \oplus R f) \rightarrow R \\
H(e, e)=H(f, f)=0, \quad H(e, f)=H(f, e)=1 .
\end{gathered}
$$


Let $u$ be a unit of $R$ such that $\bar{u}=u$. We denote by $\langle u\rangle \perp\langle-u\rangle$ the $(+1)$-Blanchfield form

$$
\begin{gathered}
(R x \oplus R y) \times(R x \oplus R y) \rightarrow R \\
x \cdot x=u, \quad y \cdot y=-u, \quad x \cdot y=y \cdot x=0 .
\end{gathered}
$$

Then we claim that $\langle u\rangle \perp\langle-u\rangle \cong H$.

To see this, note that $1=\alpha+\bar{\alpha}$ with $\alpha=1 /(1-\tau) \in R ; 1-\tau$ is a unit of $R$ because $\lambda(1)= \pm 1$.

Let $e=x+y, f^{\prime}=y$. Then $e \cdot e=0, e \cdot f^{\prime}=f^{\prime} \cdot e=-u, f^{\prime} \cdot f^{\prime}=-u$. Set $f^{\prime \prime}=f^{\prime}-\alpha e$; we have $e \cdot f^{\prime \prime}=f^{\prime \prime} \cdot e=-u$, and $f^{\prime \prime} \cdot f^{\prime \prime}=-u+\alpha u+\bar{\alpha} u=0$. Finally, let $f=-u^{-1} f^{\prime \prime}$; then $e \cdot f=f \cdot e=1$. So $\langle u\rangle \perp\langle-u\rangle$ is isometric to $H$.

Therefore $\langle 1\rangle \perp\langle-1\rangle$ is isometric to $\langle u\rangle \perp\langle-u\rangle$ for any unit $u$ of $R$ such that $\bar{u}=u$.

In order to get a counter-example to unique factorization, it suffices to find a unit $u$ such that $\langle 1\rangle \not\langle u\rangle,\langle-1\rangle \not\langle u\rangle$. Such an example is given in [(1); Section 1] for $\lambda=\phi_{15}$, the cyclotomic polynomial corresponding to the 15 th roots of unity, and $u=\tau+\tau^{-1}$.

For the (-1)-Blanchfield form case, note that in $R=\Lambda /\left(\phi_{15}\right), v=\tau-\bar{\tau}$ is a unit. Therefore $\langle v\rangle_{\perp}\langle-v\rangle$ is a $(-1)$-Blanchfield form, and we have

with $u=\tau+\tau^{-1}$.

$$
\begin{aligned}
& \langle v\rangle \perp\langle-v\rangle \cong\langle v u\rangle \perp\langle-v u\rangle \\
& \langle v\rangle ¥\langle v u\rangle, \quad\langle-u\rangle ¥\langle v u\rangle,
\end{aligned}
$$

In each case, the Alexander polynomial of the corresponding simple $(2 q-1)$-knot is $\phi_{15}^{2}$, of which the leading coefficient is 1 . Thus, as in Section 2, these knots are fibred.

\section{Non-unique factorization of definite simple $(4 q+1)$-knots, $q \geqslant 1$}

As in Section 3, it will suffice to give an example of a suitable Blanchfield form with several distinct factorizations. In fact our example will rely upon the possible nonuniqueness of factorization of the underlying knot module as a direct sum of modules. (The examples above were a little more subtle in that the underlying modules were all free as modules over the ring $\Lambda /\left(\phi_{15}\right)$, as this ring is a principal ideal domain.)

Let $\theta=13 t-25+13 t^{-1}$. The ring $R=\Lambda /(\theta)$ is isomorphic to $\mathbb{Z}\left[\gamma, \frac{1}{13}\right]$ where $\gamma=(-1+\sqrt{ }-51) / 2$ is the image of $13(t-1)$. Since $\theta\left(t^{-1}\right)=\theta(t)$, the involution of $\Lambda$ induces an involution of $R$, which is just complex conjugation, and which we denote by an overbar. Since $\theta(1)=1$, any finitely generated $R$-module which supports a nonsingular $\epsilon$-Hermitian pairing may be regarded as a knot module, and an $\epsilon$-Hermitian pairing on such a module determines an $\varepsilon$-Hermitian form via the inclusion

$$
R=\Lambda /(\theta) \cong \theta^{-1} \Lambda / \Lambda \subset \Lambda_{0} / \Lambda \text {. }
$$

Let $J$ be the $R$-ideal generated by 3 and $\sqrt{ }-51$. Then $J=\bar{J}$ and $J \bar{J}=J^{2}=(3)$, so $b_{J}(j, k)=j \bar{k} / 3$ for all $j, k$ in $J$ determines a $(+1)$-Blanchfield form on the knot module $J$. 
Let $B=b_{J} \perp b_{J}$, and let

$$
\begin{gathered}
e=((18+\sqrt{ }-51) / 13, \quad(9+\sqrt{ }-51) / 13) \\
f=((9-\sqrt{ }-51) / 13, \quad(-18+\sqrt{ }-51) / 13) .
\end{gathered}
$$

Then $B(e, e)=B(f, f)=1$ and $B(e, f)=0$, so $B$ is isometric to $b \perp b$, where $b: R \times R \rightarrow R$ is the $(+1)$-Blanchfield form on the knot module $R$ given by $b(r, s)=r \bar{s}$ for all $r, s$ in $R$. We shall show that $J$ is not a principal ideal, so that it is not isomorphic to $R$, and hence that these factorizations of $B$ are distinct.

Suppose that $J$ is principal. Then we may suppose that it is generated by an element $\alpha=A+B \gamma$ of $S=\mathbb{Z}[\gamma]$, the ring of integers of $\mathbb{Q}(\sqrt{ }-51)$, and that $\alpha$ is not divisible in $S$ by $\gamma$ or $\bar{\gamma}$, since they are units in $R$. Since 3 belongs to $J, \alpha$ divides 3 in $R$ and so divides $3 \cdot 13^{k}$ in $S$, for some large $k$. Similarly $\alpha$ divides $\sqrt{ }-51 \cdot 13^{l}$ in $S$, for some large $l$. Therefore $\alpha \bar{\alpha}=A^{2}+A B+13 B^{2}$ divides $9 \cdot 13^{2 k}$ and $51 \cdot 13^{2 l}$ in $\mathbb{Z}$, and hence divides $3 \cdot 13^{m}$ in $\mathbb{Z}$ for some large $m$. If 13 divides $\alpha \bar{\alpha}$ in $\mathbb{Z}$ then either $\gamma$ or $\bar{\gamma}$ divides $\alpha$ in $S$, since $13=\gamma \bar{\gamma}$ and $(\gamma)$ is a prime ideal as $S /(\gamma) \cong \mathbb{Z} /(13)$. As we have assumed this is not the case, $\alpha \bar{\alpha}$ must divide 3 . Since $R / J \cong \mathbb{Z} /(3), J$ is a proper ideal, and so

$$
A^{2}+A B+13 B^{2}=\alpha \bar{\alpha}=3 .
$$

This is clearly impossible and so $J$ cannot be principal. (This example was discussed in greater detail in (5), where it was indicated how other examples with knot module annihilated by an irreducible knot polynomial $\delta$ might be sought whenever $\delta$ is such that $\delta=\bar{\delta}$ and $\Lambda /(\delta)$ contains a non-principal ideal $I$ such that $I \bar{I}$ is principal.)

Since any real quadratic space of rank 2 with an isometry whose characteristic polynomial has complex roots (such as $\theta(t)$ ) must be definite, any simple $(4 q+1)$-knot with Blanchfield form $B=b \perp b=b_{J} \perp b_{J}$ is a definite knot with two distinct factorizations into irreducible knots.

\section{Non-unique factorization of definite simple $(4 q-1)$-knots, $q \geqslant 2$}

As in Sections 3 and 4, it suffices to show that factorization is not unique for definite $(-1)$-Blanchfield forms. Let $\lambda(t)=53 t^{8}-105 t^{4}+53$. Then $\lambda$ is irreducible over $\mathbb{Q}$ (this can be checked by computing the roots of $\lambda$ ). Let

$$
K=\mathbb{Q}[t] /(\lambda)=\mathbb{Q}(\tau), \quad R=\mathbb{Z}\left[t, t^{-1}\right] /(\lambda)=\mathbb{Z}\left[\tau, \tau^{-1}\right],
$$

where $\tau$ is a root of $\lambda$. Note that $R$ is integrally closed by ((13), Theorem 28.2, p. 93).

We shall follow the same idea as in Section 4 . We shall begin by constructing a non-principal ideal $I$ of $R$. We have

$$
N_{K / Q}(1-\tau)=\frac{1}{53} \lambda(1)=\frac{1}{53} \in R .
$$

Therefore 53 is a unit of $R$. As $N_{K / Q}\left(1-\tau^{4}\right)=1 / 53^{4}, 1-\tau^{4}$ is also a unit of $R$. Let $\omega=\left(1-\tau^{4}\right)^{-1} \in R$, and let $I$ be the $R$-ideal generated by 5 and $\omega+1$.

Claim 1. $I$ is not principal.

Proof. Let $K_{1}=\mathbb{Q}[t] /\left(53 t^{2}-105 t+53\right)=\mathbb{Q}\left(\tau^{4}\right), R_{1}=\mathbb{Z}\left[\tau^{4}, \tau^{-4}\right]$. Then $\omega \in R_{1}$. Let $I_{1}=(5, \omega+1)$. It is straightforward to check that $I_{1}$ and $I_{1}^{2}$ are not principal (use the 
same method as in Section 4), and that $I_{1}^{3}=(\omega-9) R_{1}$ : therefore $I_{1}$ is of order 3 . (In fact, it suffices to prove that $I_{1}$ is not principal. The table on p. 101 of (13) implies that $I_{1}$ is then of order 3.)

Let $K_{2}=\mathbb{Q}[t] /\left(53 t^{4}-105 t^{2}+53\right)=\mathbb{Q}\left(\tau^{2}\right), R_{2}=\mathbb{Z}\left[\tau^{2}, \tau^{-2}\right]$, and let $I_{2}=(5, \omega+1)$ be the extension of $I_{1}$ to $R_{2}$. Using ((13), Section 29, p. 95) we see that $R_{1}$ and $R_{2}$ are integrally closed. We have: $R \cap K_{2}=R_{2}, R_{2} \cap K_{2}=R_{1} . I$ is the extension of $I_{2}$ to $R$.

The following lemma shows that $I_{2}$ and $I$ are also non-principal, of order 3 .

Lemma 5.1. Let $E / F$ be a quadratic extension of number fields, let $A$ be an integrally closed subring of $E$ which is sent into itself by Gal $(E / F)$, and let $B=A \cap F$. Let b be an ideal of $B$ such that $a=b A$ is principal. Then $b^{2}$ is principal.

Proof: Let $\sigma: E \rightarrow E$ generate Gal $(E / F)$. We have $a=b A$, therefore $\sigma(a)=a$. But $a$ is principal by hypothesis, so there exists an $x \in E$ such that $a=x A$. Then

$$
a^{2}=a \cdot \sigma(a)=x \cdot \sigma(x) A .
$$

Let $y=x \cdot \sigma(x)$. We have $\sigma(y)=y$, so $y \in F$. Then $b^{2}=a^{2} \cap F=y B$, so $b^{2}$ is principal.

Let - denote $\mathbb{Q}$-involution of $K$ which sends $\tau$ to $\tau^{-1}$. Then

$$
I \bar{I}=(25,5(\omega+1), \quad 5(\bar{\omega}+1), 55)=5 R .
$$

Let $b_{I}: I \times I \rightarrow R$ be given by $b_{I}(x, y)=x \bar{y} / 5$. Then $b_{I}$ is a $(+1)$-Blanchfield form.

Let $L=I \oplus I \oplus I$, and define $b_{L}: L \times L \rightarrow R$ to be $b_{L}=b_{I} \perp b_{I} \perp b_{I}$.

Let $b: R \times R \rightarrow R$ be given by $b(x, y)=53 x \bar{y}$. As 53 is a unit of $R, b$ is a $(+1)$-Blanchfield form.

Claim 2. $b$ is an orthogonal summand of $b_{L}$.

Proof. Let $e=(10+(\omega+1), \omega+1,5) \in L$. Direct computation gives $b_{L}(e, e)=53$. Then $R e$ is a submodule of $L$ such that $b_{L} \mid(R e \times R e) \cong b$ is unimodular, therefore $b$ is an orthogonal summand of $b_{L}$.

So $b_{L}=b_{I} \perp b_{I} \perp b_{I} \cong b \perp b^{\perp}$, and $b_{I} ¥ b$ because $I$ is not principal by Claim 1 . Thus we have proved that the $(+1)$-Blanchfield form $b_{L}$ has at least two nonequivalent factorizations.

Now we shall change $b_{L}$ in order to get a definite (-1)-Blanchfield form.

Claim 3. There exists an $R$-ideal $J$ and a (-1)-Blanchfield form $B: J \times J \rightarrow R$ such that $B \otimes_{R} b_{I}: J I \times J I \rightarrow R$ is a definite (-1)-Blanchfield form.

Claim 3 implies the non-uniqueness of the factorization of definite ( -1$)$-Blanchfield forms. Indeed,

$$
B \otimes b_{L}=B \otimes b_{I} \perp B \otimes b_{l} \perp B \otimes b_{I} \simeq B \otimes b \perp B \otimes b^{\perp} .
$$

We have seen that $I$ is non-principal, therefore $I J$ is not isomorphic to $J$. So we have $B \otimes b_{1} \approx B \otimes b$.

Proof of Claim 3. Let $\alpha=(1-\tau)^{-1} \in R$. Let $\phi(t)=t^{8} \lambda\left(1-t^{-1}\right) \in \mathbb{Z}[t]: \phi$ is the minimal polynomial of $\alpha$. We have

$$
\phi(t)=t^{8}-4 t^{7}+854 t^{6}-2548 t^{5}+3605 t^{4}-53 \cdot 56 t^{3}+53 \cdot 28 t^{2}-8 \cdot 53 t+53 .
$$


Let

$$
\delta=\phi^{\prime}(\alpha)(1-2 \alpha)=-3388 \alpha^{3}(1-\alpha)^{3}+106.56 \alpha^{2}(1-\alpha)^{2}-53 \cdot 56 \alpha(1-\alpha)+8 \cdot 53 .
$$

Let $F=\{x \in K: \bar{x}=x\}$. $F$ has 4 real embeddings $\sigma_{1}, \sigma_{2}, \sigma_{3}, \sigma_{4}$. It is straightforward to check that $\delta$ is positive at two of these embeddings, say $\sigma_{1}$ and $\sigma_{2}$, and negative at $\sigma_{3}$ and $\sigma_{4}$.

Let $\beta=(\tau-\bar{\tau})^{2} \in F$ : notice that $K=F(\tau-\bar{\tau})$. Denote the Hilbert symbol by $(,)_{P}$.

There exists an $a \in F$ such that

$$
(a, \beta)_{P}= \begin{cases}-1 & \text { if } P=\sigma_{3}, \sigma_{4} \\ +1 & \text { otherwise (that is, for } P=\sigma_{1}, \sigma_{2}, \text { or a discrete prime) }\end{cases}
$$

To see this, apply Theorem 71:19and Corollary $71: 19 a$ of (15) with $T=\left\{\sigma_{3}, \sigma_{4}\right\}$. Notice that $\beta=-\langle\tau-\bar{\tau}) \overline{(\tau-\bar{\tau})}$, so $\beta$ is negative at all real embeddings of $F$.

As $(a, \beta)_{P}=+1$ for $P$ discrete, there exists an $R$-ideal $J$ such that $B^{\prime}: J \times J \rightarrow R$ given by $B^{\prime}(x, y)=a x, \bar{y}$ is a $(+1)$-Blanchfield pairing (see ((13), lemma $2 \cdot 4 \cdot 3$, p. 81)).

Now $1-2 \alpha$ is a unit of $R$. Indeed, $1-2 \alpha=(\tau+1) /(\tau-1)$, and

$$
N_{K / Q}(\tau+1)=N_{K / Q}(\tau-1)=1 / 53, \text { so } N_{K / Q}(1-2 \alpha)=1 \text {. }
$$

Define $B: J \times J \rightarrow R$ by $B(x, y)=(1-2 \alpha)^{-1} a x, \bar{y}$. We have seen that $1-2 \alpha$ is a unit, and clearly $\overline{1-2 \alpha}=-(1-2 \alpha)$, therefore $B$ is a $(-1)$-Blanchfield pairing.

It remains to prove that $h=B \otimes b_{I}$ is definite. To see this, it suffices to show that the extension $h_{K}$ of $h$ to $K$ is definite. We have

$$
h_{K}: K \times K \rightarrow K, h_{K}(x, y)=(1-2 \alpha)^{-1} a x, \bar{y} / 5 .
$$

Since $K=\mathbb{Q}(\alpha)$, we can write $x \in K$ in the form

$$
x=\sum_{i=0}^{7} x_{i} \alpha^{i}, x_{i} \in \mathbb{Q}
$$

and this expression is unique. Define $s: K \rightarrow \mathbb{Q}$ by $s(x)=x_{7}$, as in ((21), p. 239). We have $s(x)=\operatorname{Tr}_{K / \mathbb{Q}}\left(x / \phi^{\prime}(\alpha)\right)$. (See (21), p. 239), and $s\left(h_{K}(x, y)\right)=S(x, y)$, where $S^{-1}$ is the rational intersection form corresponding to $h_{K}$ (see (21) and (20): Section 2)).

By definition, $h_{K}$ is definite if and only if $S^{-1}$ is a definite quadratic form. Clearly $S^{-1}$ is definite if and only if $S$ is definite. We have

$$
S(x, x)=s\left(h_{K}(x, x)\right)=\operatorname{Tr}_{K / Q}(a x, \bar{x} / 5 \delta)>0
$$

if $x \neq 0$, (recall that $\delta=\phi^{\prime}(\alpha)(1-2 \alpha)$ ) because $a / \delta$ is totally positive by construction, and $x \bar{x}$ is also totally positive as the involution becomes complex conjugation at every $\mathbb{C}$-embedding of $K$.

Therefore $S(x, x)>0$ if $x \neq 0$, so $S$ is positive definite.

\section{Non-unique factorization of 3-knots}

Let $k$ be a simple 3-knot, with Seifert matrix $S$-equivalent to the non-singular matrix $A$. If $A$ is unimodular, then we say that $k$ is algebraically fibred. (If $k$ were a simple $(4 q-1)$-knot, $q>1$, then $k$ would be fibred by the Browder-Levine theorem (2).) 
Consider the following matrices.

$$
\begin{aligned}
& B=\left[\begin{array}{rrrrrrrr}
1 & 0 & -1 & 0 & 0 & 0 & 0 & 0 \\
0 & 1 & 0 & -1 & 0 & 0 & 0 & 0 \\
0 & 0 & 1 & -1 & 0 & 0 & 0 & 0 \\
0 & 0 & 0 & 1 & -1 & 0 & 0 & 0 \\
0 & 0 & 0 & 0 & 1 & -1 & 0 & 0 \\
0 & 0 & 0 & 0 & 0 & 1 & -1 & 0 \\
0 & 0 & 0 & 0 & 0 & 0 & 1 & -1 \\
0 & 0 & 0 & 0 & 0 & 0 & 0 & 1
\end{array}\right] \\
& C=\left[\begin{array}{rrrrrrrr}
1 & -1 & -2 & -3 & -3 & -3 & -2 & -1 \\
2 & 1 & -1 & -2 & -3 & -3 & -3 & -2 \\
3 & 2 & 1 & -1 & -2 & -3 & -3 & -3 \\
3 & 3 & 2 & 1 & -1 & -2 & -3 & -3 \\
3 & 3 & 3 & 2 & 1 & -1 & -2 & -3 \\
2 & 3 & 3 & 3 & 2 & 1 & -1 & -2 \\
1 & 2 & 3 & 3 & 3 & 2 & 1 & -1 \\
0 & 1 & 2 & 3 & 3 & 3 & 2 & 1
\end{array}\right]
\end{aligned}
$$

Each matrix possesses the following properties:

(i) $\operatorname{det}\left(A+A^{\prime}\right)=1$;

(ii) signature $\left(A+A^{\prime}\right)=8$.

In addition we have

(iii) $\operatorname{det}\left(t B+B^{\prime}\right)=1+t-t^{3}-t^{4}-t^{5}+t^{7}+t^{8}=\phi_{30}(t)$;

(iv) $\operatorname{det}\left(t C+C^{\prime}\right)=1-t+t^{3}-t^{4}+t^{5}-t^{7}+t^{8}=\phi_{15}(t)$.

By (12) there exist unique 3-knots $k, l, m$ with Seifert matrices $S$-equivalent to

$$
\left(\begin{array}{ll}
B & 0 \\
0 & \mathrm{~B}
\end{array}\right),\left(\begin{array}{ll}
C & 0 \\
0 & C
\end{array}\right),\left(\begin{array}{ll}
B & 0 \\
0 & C
\end{array}\right)
$$

respectively. By (12), we have $k+l=m+m$. Because the signature of a 3 -knot must be divisible by 16 (see (12)), it is clear that each of the knots $k, l, m$ is irreducible. All three knots are distinguished by their Alexander polynomials, these being $\phi_{30}(t) \phi_{30}(t)$, $\phi_{15}(t) \phi_{15}(t), \phi_{30}(t) \phi_{15}(t)$ respectively. Finally, the knot $k+l$ is definite and algebraically fibred. Thus the analogue of Theorem $2 \cdot 1$ fails for 3 -knots.

\section{Unique factorization of odd semisimple finite $2 q-k n o t s, q \geqslant 4$}

In this section we shall sketch a proof of the following theorem, given in (6).

THEOREM 7-1. If $k$ is an odd simple $2 q-k n o t, q \geqslant 4$, whose knot module $H_{q}(\widetilde{K} ; \mathbb{Z})$ is semisimple and such that either $q$ is even or $t+1$ acts invertibly, then $k$ has a unique factorization into irreducible knots.

By means of Kojima's classification of odd simple $2 q$-knots, $q \geqslant 4$, we may reduce the proof of this theorem to an argument about the factorization of certain $\epsilon$-Levine pairings (for $\epsilon=(-1)^{q+1}$ ). We shall first explain the term 'semisimple'. 
A finite $\Lambda$-module $M$ is the direct sum of its localizations $M$ at the various maximal ideals $m$ of $\Lambda$. The localization $M_{m}$ may be regarded as a module over the $m$-adic completion $\Lambda_{\hat{m}}=\lim \Lambda / m^{n}$. Let $m=(p, g(t))$ where $p$ is a rational prime and $g(t)$ is a monic polynomial in $\mathbb{Z}[t]$ whose image in $\mathbb{Z} / p \mathbb{Z}[t]$ is irreducible. Then the completion $\Lambda_{\hat{m}}$ is isomorphic to $S[[T]]$ where $S=\mathbb{Z}_{\hat{p}}[\xi]$ is an unramified extension of the $\boldsymbol{p}$-adic integers $\mathbb{Z}_{\hat{p}}$, generated by a root of unity $\xi$ such that $g(\xi) \equiv 0$ modulo $(p)$, and where $t$ has image $\xi(1-T)$. (That such an isomorphism exists is a srecial case of I.S. Cohen's structure theorem for complete regular local rings ((17): p. V-16).) The localization $M$ is semisimple if $T \cdot M_{m}=0$; the module $M$ is semisimple if each such localization is semisimple. Semisimple modules may be recognized by the following criterion. A finite $\Lambda$-module $M$ is semisimple if and only if $\operatorname{Ann} M=\Pi_{i=1}^{r}\left(p_{i}^{e_{i}}, g_{i}\right)$ where $p_{i}$ is a rational prime and $g_{i}$ is congruent modulo $\left(p_{i}^{e_{i}}\right)$ to an irreducible factor of a cyclotomic polynomial in $\mathbb{Z} / p_{i}^{e_{i}}[t]$ for $1 \leqslant i \leqslant r$, and where the maximal ideals $\left(p_{i}, g_{i}\right)$ are all distinct. (This fact is not used in proving the theorem.)

Suppose that the finite knot module $M$ supports an $\epsilon$-Levine pairing

$$
[,]: M \times M \rightarrow \mathbb{Q} / \mathbb{Z} \text {. }
$$

Then the localizations $M_{m}$ and $M_{n}$ are orthogonal unless $n=\bar{m}$. If $m \neq \bar{m}$ the pairing on $M_{m} \oplus M_{\bar{m}}$ is determined uniquely by the module structure of $M_{m}$. If $m=\bar{m}$ then the involution of $\Lambda$ induces involutions on $\Lambda_{\hat{m}}$ and $S$ mapping $\xi$ to $\xi^{-1}$ and $T$ to $T /(T-1)$, and the pairing $[$,$] determines a pairing$

$$
\{,\}: M_{\hat{m}} \times M_{\hat{m}} \rightarrow S \otimes \mathbb{Q} / \mathbb{Z}=S_{0} / S
$$

which is non-singular, $S$-linear in its first argument, $\epsilon$-conjugate symmetric

and such that

$$
(\{n, m\}=\epsilon\{\overline{m, n}\} \text { for all } m, n \text { in } M)
$$

$$
\{T m, n\}=\{m,(T /(T-1)) n\} \text { for all } m, n \text { in } M \text {. }
$$

If now $M$ is assumed semisimple each localization $M_{m}$ is a module over a discrete valuation ring $S=\Lambda_{\hat{m}} /(T)$, and so has an essentially unique factorization as a direct sum of (irreducible) cyclic modules. By the remarks above we may assume that $M$ is annihilated by some power of a maximal ideal $m$ such that $\bar{m}=m$.

An $\epsilon$-Levine pairing on such a module is equivalent to a non-singular $\epsilon$-conjugate symmetric pairing into $S_{0} / S$ which is $S$-linear in its first argument. For brevity, we shall refer to such a pairing on a finite $S$-module as an ( $\epsilon$-torsion) form. We recall that $S=\mathbb{Z}_{\hat{p}}[\xi]$ where $\xi$ is a root of unity which is not congruent to 1 modulo $(p)$ (since $M$ is a knot module). The extension $S / \mathbb{Z}_{\hat{p}}$ is unramified, so the unique maximal ideal of $S$ is generated by $p$, and $S$ has an involution which maps $\xi$ to $\bar{\xi}=\xi^{-1}$. The involution is the identity if and only if $\xi=-1$, and in this case $p$ must be odd.

Let $\epsilon-S\left(p^{k}\right)$ denote the $\epsilon$-torsion form with underlying module $S /\left(p^{k}\right)$, generated by $e=1+\left(p^{k}\right)$, and with pairing determined by

$$
\begin{aligned}
& \{e, e\}=1 / p^{k} \text { if } \epsilon=+1 \\
& \{e, e\}=(\xi-\bar{\xi}) / p^{k} \text { if } \epsilon=-1 \text { and the involution is nontrivial. }
\end{aligned}
$$

(If $\epsilon=-1, p$ is odd, and the involution is trivial then there is no cyclic $\epsilon$-torsion form.) 
Proposition 7.2. If the invalution on $S$ is nontrivial then any $\epsilon$-torsion form $M,\{$,$\} is$ an orthogonal direct sum of copies of $\epsilon-S\left(p^{i}\right)$, for various $j \geqslant 1$.

CoROLLARY 7.3. If the involution on $S$ is nontrivial, then any $\epsilon$-torsion form is determined up to isometry by its underlying module, and has an essentially unique decomposition into irreducible forms.

Now let us suppose that the involution on $S$ is trivial, so that $\xi=-1, S=\mathbb{Z}_{\hat{p}}$ and $p$ is odd. Let $r$ be the smallest positive integer which is not congruent to a square modulo $(p)$. (In fact we could use any non-quadratic residue instead of $r$.) Let

$$
\widetilde{+1}-S /\left(p^{k}\right)
$$

denote the +1 -torsion form over $S$ whose underlying module is $S /\left(p^{k}\right)$, generated by $f=1+\left(p^{k}\right)$, and with pairing determined by $\{f, f\}=r / p^{k}$. Let $H_{k}$ denote the -1 -torsion form over $S$ whose underlying module is $\left(S /\left(p^{k}\right)\right)^{2}$, generated by $h$ and $h^{\prime}$, and with pairing determined by $\left\{h, h^{\prime}\right\}=1 / p^{k}$.

Propositron 7.4. If the involution on $S$ is trivial then any +1-torsion form $M,\{$, is an orthogonal direct sum of copies of $+1-S /\left(p^{j}\right)$ and $\widetilde{+1}-S /\left(p^{j}\right)$ for various $j \geqslant 1$; moreover $+1-S /\left(p^{i}\right)$ and $\widetilde{+} 1-S /\left(p^{i}\right)$ are distinct, but $\left(+1-S /\left(p^{i}\right)\right) \oplus\left(+1-S /\left(p^{i}\right)\right)$ is isomorphic to $\left(\widetilde{+1}-S /\left(p^{j}\right)\right) \oplus\left(\widetilde{+1}-S /\left(p^{j}\right)\right)$ for each $j \geqslant 1$. Any -1 -torsion form is an orthogonal direct sum of copies of $H_{j}$ for various $j$; moreover $H_{j}$ is irreducible.

CoRollary 7.5. If the involution on $S$ is trivial, any-1-torsion form is determined by its underlying module, and has an essentially unique decomposition into irreducible forms. I

Let $M,\{$,$\} be a +1$-torsion form whose underlying module is freely generated over $S /\left(p^{k}\right)$ by the elements $m_{1}, \ldots, m_{d}$ with $d \geqslant 1$, and suppose that $\left\{m_{i}, m_{j}\right\}=S_{i j} / p^{k}$ for some element $S_{i j}$ in $S$ (not necessarily a unit). Let DET $\{$,$\} be the image of \operatorname{det}\left[S_{i j}\right]$ in $\left(S /\left(p^{k}\right)\right)^{*} /\left(\left(S /\left(p^{k}\right)\right)^{*}\right)^{2}=\mathbb{Z} / 2 \mathbb{Z}$.

COROLLARY 7.6. There are up to isomorphism two +1 -torsion forms on a non-trivial free $S /\left(p^{k}\right)$-module $M$ distinguished by the value of DET $\{$,$\} . Each of these factors is$ an orthogonal direct sum of cyclic forms and the number of essentially distinct such factorizations is the number of factorizations of DET $\{$,$\} as a product of d$ elements in the group $\mathbb{Z} / 2 \mathbb{Z}$ (where $d$ is the minimal number of generators of $M$ ).

Theorem $7 \cdot 1$ is an immediate consequence of Corollary $7 \cdot 3$ and Corollary $7 \cdot 5$.

\section{Non-unique factorization of odd finite $2 q-k n o t s, ~ q \geqslant 4$.}

Corollary $7.6 \mathrm{implies}$ that for each odd $q \geqslant 5$ there is an odd finite $2 q$-knot $k$ with $H_{q}(\tilde{K} ; \mathbb{Z})$ semisimple and which has more than one factorization into irreducible knots. The example given in (1) (for $q$ odd) is of this nature, having knot module isomorphic to $(\Lambda /(5, t+1))^{2}$. There is only one maximal ideal to consider, and we may take $p=5$ and $\xi=-1$. The involution is trivial, and $(\Lambda /(5, t+1))^{2}$ admits one $(+1)$-torsion form with DET $=[ \pm 1]$, the class of a square, and one with DET $=[ \pm 2]$, the class of a nonsquare. The example of (1) is the first of these, and has two factorizations as a direct sum of two cyclic forms since $[ \pm 1]=[ \pm 1]^{2}=[ \pm 2]^{2}$; the second has unique factorization since $[ \pm 2]=[ \pm 1][ \pm 2]$. 
Uniqueness of factorization can also fail for an odd simple $2 q$-knot for each even $q \geqslant 4$, but no such knot can have semisimple knot module. The example given for this case in (1) is as follows. Let $e$ be a fixed generator for the cyclic module $E=\Lambda /\left(5,(t+1)^{2}\right)$ and let [, ] and [, ] be the (-1)-Levine pairings on $E$ determined by $[e, t e]=\frac{1}{5} \bmod \mathbb{Z}$ and $[e, t e]^{\prime}=\frac{2}{5} \bmod \mathbb{Z}$ respectively. Suppose that $\phi: E \rightarrow E$ is an isometry from [,] to $\pm[,]^{\prime}$, sending $e$ to $\phi(e)=a e+b t e$ with $a, b$ in $\mathbb{Z}$. Then, $\bmod \mathbb{Z}$,

$$
\begin{aligned}
\frac{1}{\mathbf{5}} & =[e, t e] \\
& = \pm[\phi(e), \phi(t e)]^{\prime} \\
& = \pm\left[a e+b t e, a t e+b t^{2} e\right]^{\prime} \\
& = \pm[a e+b t e, a t e-b e-2 b t e]^{\prime} \\
& = \pm\left([a e,(a-2 b) t e]^{\prime}+[b t e,-b e]^{\prime}\right) \\
& = \pm\left(a^{2}-2 a b+b^{2}\right) \cdot \frac{2}{5},
\end{aligned}
$$

which implies that \pm 2 is a perfect square modulo 5 , which is false. Therefore [, ] is not isometric to either $[,]^{\prime}$ or $-[,]^{\prime}$. by

But the map $\Phi: E^{2} \rightarrow E^{2}$, given in matrix form with respect to the basis $\{(e, 0),(0, e)\}$

$$
\left(\begin{array}{rr}
2 & 2 \\
-2 & 2
\end{array}\right)
$$

is an isometry between $[,] \perp-[$,$] and [,]^{\prime} \perp-[,]^{\prime}$. Thus there is a $(-1)$-Levine pairing on the finite knot module $E^{2}=\left(\Lambda /\left(5,(t+1)^{2}\right)\right)^{2}$ which has more than one factorization as a sum of irreducible pairings. Of course the underlying knot module is not semisimple, as $T=t+1$ does not act as the zero endomorphism.

\section{REFERENCES}

(1) BAYER, E. Factorisation is not unique for higher dimensional knots. Comm. Math. Helv. 55 (1980), 583-592.

(2) Browder, W. and Levine, J. Fibering manifolds over a circle. Comm. Math. Helv. 40 (1965), 153-160.

(3) Cassels, J. W. S. Rational quadratic forms. Academic Press (1978). LMS monograph no. 13.

(4) Crowell, R. The group $G^{\prime} / G^{\prime \prime}$ of a knot group $G$. Duke Math. Jour. 30 (1963), 349-354.

(5) Hrtlman, J. A. Blanchfield pairings with squarefree Alexander polynomials. Math. Zeit. 176 (1981), 551-563.

(6) Hillman, J. A. Finite knot modules and the factorisation of certain simple knots. Math. Ann. (to appear).

(7) Kearton, C. Classification of simple knots by Blanchfield duality. Bull. Amer. Math. Soc. 79 (1973), 952-955.

(8) Kearton, C. Blanchfield duality and simple knots. Trans. Amer. Math. Soc. 202 (1975), $141-160$.

(9) Kervarre, M. A. Les nœuds de dimensions supérieures. Bull. Soc. Math. France. 93 (1965), 225-271.

(10) Kojima, S. Classification of simple knots by Levine pairings. Comm. Math. Helv. 54 (1979), $356-367$.

(11) LeviNe, J. Unknotting spheres in codimension two. Topology 4 (1965), 9-16.

(12) Levine, J. An algebraic classification of some knots of codimension two. Comm. Math. Helv. 45 (1970), 185-198.

(13) LeviNe, J. Algebraic structure of knot modules (Springer, Lecture Notes in Mathematics, no. $772(1980))$. 
(14) Maeda, T. On a composition of knot groups. II. Algebraic bridge index. Mathematics Seminar Notes Kobe University 5 (1977), 457-464.

(15) O'Meara, O. T. Introduction to quadratic forms. (Springer, 1971).

(16) Schubert, H. Die eindeutige Zerlegbarkeit eines knotens in Primknoten. S.-B. Heidel. berger Akad. Wiss. Math. Nat. Kl. 3 (1949), 57-104.

(17) Serne, J. P. Algèbre locale-multiplicités (Springer, Lecture Notes in Mathematics, no. 11, 1965).

(18) SosinskiI, A. B. Decomposition of knots. Math. USSR-Sb. 10 (1970), 139-150.

(19) Trotser, H. F. Homology of group systems with applications to knot theory. Annals of Math. 76 (1962), 464-498.

(20) Trotwer, H. F. On S-equivalence of Seifert matrices. Invent. Math. 20 (1973), 173-207.

(21) Trotrer, H. F. Knot modules and Seifert matrices (Springer, Lecture Notes in Mathematics, no. 685, Knot Theory (1978)), pp. 291-299. 